\title{
ON THE GENERAL AND MEASURABLE SOLUTIONS OF SOME FUNCTIONAL EQUATIONS
}

\author{
Prem Nath, Dhiraj Kumar Singh*
}

\begin{abstract}
The general solutions of two functional equations, without imposing any regularity condition on any of the functions appearing, have been obtained. From these general solutions, the Lebesgue measurable solutions have been deduced by assuming the function(s) to be measurable in the Lebesgue sense.
\end{abstract}

\section{Introduction}

For $n=1,2, \ldots$, let $\Gamma_{n}=\left\{\left(p_{1}, \ldots, p_{n}\right): p_{i} \geq 0, i=1, \ldots, n ; \sum_{i=1}^{n} p_{i}=1\right\}$ be the set of all $n$-component complete discrete probability distributions with nonnegative elements. Let $\mathbb{R}$ denote the set of all real numbers; $I=\{x \in \mathbb{R}$ : $0 \leq x \leq 1\}=[0,1]$, the closed unit interval; $] 0,1[=\{x \in \mathbb{R}: 0<x<1\}$, the open unit interval and $] 0,1]=\{x \in \mathbb{R}: 0<x \leq 1\}$. Below, we mention three functional equations

$$
\begin{aligned}
& f(p q)=q f(p)+p f(q), \\
& f(p q)=q^{\alpha} f(p)+p^{\alpha} f(q)
\end{aligned}
$$

${ }^{*}$ Corresponding author.

Received: 15.09.2016. Accepted: 16.05.201\%. Published online: 05.08.2017.

(2010) Mathematics Subject Classification: 39B22, 39B52.

Key words and phrases: logarithmic function, multiplicative function, function measurable in the Lebesgue sense, continuous function, stability of a functional equation, the Shannon entropy. 
and

$$
f(p q)=q^{\beta} f(p)+p^{\alpha} f(q),
$$

where $f: I \rightarrow \mathbb{R}$ is an unknown function, $p, q \in I ; \alpha$ and $\beta$ are fixed positive real exponents which satisfy the following conventions

$$
0^{\alpha}:=0, \quad 1^{\alpha}:=1, \quad 0^{\beta}:=0, \quad 1^{\beta}:=1 .
$$

Let us consider (1.1). In this equation let us replace $p$ by $p_{i}, q$ by $q_{j}$ and sum both sides of the resulting equations with respect to $i=1, \ldots, n ; j=1, \ldots, m$ such that $\left(p_{1}, \ldots, p_{n}\right) \in \Gamma_{n},\left(q_{1}, \ldots, q_{m}\right) \in \Gamma_{m}, n$ and $m$ being positive integers, we get the functional equation

$$
\sum_{i=1}^{n} \sum_{j=1}^{m} f\left(p_{i} q_{j}\right)=\sum_{i=1}^{n} f\left(p_{i}\right)+\sum_{j=1}^{m} f\left(q_{j}\right)
$$

which is due to T.W. Chaundy and J.B. Mcleod [2]. The functional equation (1.5) is useful in characterizing the Shannon entropies (see [10]) $H_{n}: \Gamma_{n} \rightarrow \mathbb{R}$, $n=1,2, \ldots$ defined as

$$
H_{n}\left(p_{1}, \ldots, p_{n}\right)=-\sum_{i=1}^{n} p_{i} \log _{2} p_{i}
$$

for all $\left(p_{1}, \ldots, p_{n}\right) \in \Gamma_{n}$ with $0 \log _{2} 0:=0$. This shows the importance of $(1.1)$ in information theory.

J. Tabor ([11, p. 172]) has discussed the stability of (1.1) in a somewhat broader context, by taking $f:] 0,1] \rightarrow E$ where $E$ is a Banach space and also assuming some additional conditions.

The functional equation 1.2 with $p \in I$ (or $] 0,1[$ or $] 0,1]$ ), $q \in I$ (or ]0,1[ or $] 0,1]$ ) and sometimes using different symbols in place of $f$ appears in ([3, p. 349]), ([5, p. 130]), ([6, p. 266]), ([4, p. 41]), ([7, p. 107]) and ([8, p. 3]). The utility of 1.2 in stability theory concerning functional equations has also been pointed out ([7, p. 107]).

The functional equation (1.3) appears as equation (36) on p. 166 in the book, by M. Behara [1], which seems to have escaped the attention of many researchers working on various entropies in information theory. The object of this paper is to study the functional equations

$$
f(p q)=q^{\beta} f(p)+p^{\alpha} f(q)+c f(p) f(q)
$$


and

$$
f(p q)=q^{\beta} f(p)+p^{\alpha} f(q)+g(p) f(q)
$$

where $f: I \rightarrow \mathbb{R}, g: I \rightarrow \mathbb{R}$ are unknown functions; $p, q \in I ; \alpha$ and $\beta$ are fixed positive real exponents which satisfy the conventions stated in (1.4); and $c$ is a given real constant.

If $c=0$, then (FE1) reduces to $(1.3)$. On the other hand, if $g(p) \equiv 0$, then (FE2) reduces to (1.3). Equation (1.3) has motivated us to study (FE1) and (FE2).

The plan of this paper is as follows: In section 2, we present known definitions and results which will be used in the subsequent sections. Section 3 is devoted to determining the general solutions of (FE2) and (FE1) (with $c \neq 0$ ) without imposing any regularity restriction on the functions $f, g$ in (FE2) and function $f$ in (FE1). Section 4 is devoted to determining all Lebesgue measurable solutions of $($ FE2 $)$ and FE1) (with $c \neq 0)$.

\section{Some known definitions and results}

We need the following:

Definition 2.1. A function $M: I \rightarrow \mathbb{R}$ is said to be multiplicative if $M(p q)=M(p) M(q)$ for all $p, q \in I$.

Definition 2.2. A function $\ell: I \rightarrow \mathbb{R}$ is said to be logarithmic if $\ell(0)=0$ and

$$
\ell(p q)=\ell(p)+\ell(q)
$$

for all $p, q \in] 0,1]$.

ReSult 2.1 ([1]). Suppose a function $f: I \rightarrow \mathbb{R}$ satisfies equation (1.3) for all $p, q \in I ; \alpha$ and $\beta$ being fixed positive real exponents which satisfy the conventions in (1.4). The following conclusions hold:

(I) If $\beta \neq \alpha$, then (for all $p \in I$ )

$$
f(p)=\lambda\left(p^{\alpha}-p^{\beta}\right)
$$

where $\lambda$ is an arbitrary real constant (which may depend upon $\alpha$ and $\beta$ ). 
(II) If $\beta=\alpha$, then (for all $p \in I$ )

$$
f(p)=p^{\alpha} \ell(p)
$$

where $\ell: I \rightarrow \mathbb{R}$ is a logarithmic function in the sense of Definition 2.2 and $0^{\alpha} \ell(0)=0$.

For the proof of this result, see the proof of Theorem 2, pages 165-168, in [1].

\section{On the functional equations $(\overline{F E} 2$ and (FE1)}

In this section, we determine all general solutions of the functional equations $($ FE2 $)$ and $($ FE1) (with $c \neq 0$ ) without imposing any regularity condition on any of the functions $f$ and $g$.

THEOREM 3.1. If the functions $f: I \rightarrow \mathbb{R}, g: I \rightarrow \mathbb{R}$ with $f \neq 0$, satisfy the functional equation (FE2) for all $p, q \in I, \alpha$ and $\beta$ being positive real exponents which satisfy the conventions stated in (1.4), then any general solution $(f, g)$ of (FE2) has one of the forms (for all $p \in I$ )

$$
\begin{array}{r}
\beta \neq \alpha, \quad f(p)=\lambda\left(p^{\alpha}-p^{\beta}\right), \quad g(p) \equiv 0, \\
\beta=\alpha, \quad f(p)=p^{\alpha} \ell(p), \quad g(p) \equiv 0, \\
f(p)=\bar{\mu}\left[M(p)-p^{\beta}\right], \quad g(p)=\left[M(p)-p^{\alpha}\right], \\
\beta \neq \alpha, \quad f(p)=p^{\beta} \ell(p), \quad g(p)=\left(p^{\beta}-p^{\alpha}\right),
\end{array}
$$

for some constants $\lambda \neq 0$ and $\bar{\mu} \neq 0 ; \ell: I \rightarrow \mathbb{R}$ is logarithmic $(\ell \neq 0)$ and $M: I \rightarrow \mathbb{R}$ is multiplicative. Furthermore, $0^{\alpha} \ell(0):=0,0^{\beta} \ell(0):=0$; and the restriction of $M$ to the open interval ]0,1[is not identical to either $p \mapsto p^{\alpha}$ or $p \mapsto p^{\beta}$.

To prove this theorem, we need the following:

LEMMA 3.2. If a function $g: I \rightarrow \mathbb{R}$ satisfies the functional equation

$$
g(p q)=q^{\alpha} g(p)+p^{\alpha} g(q)+g(p) g(q)
$$


for all $p, q \in I$ and $\alpha$ is a fixed positive real exponent such that $0^{\alpha}:=0$ and $1^{\alpha}:=1$, then any general solution $g$ of (FE3) has one of the forms (for all $p \in I)$

$$
\begin{aligned}
& g(p) \equiv 0, \\
& g(p)=M(p)-p^{\alpha},
\end{aligned}
$$

where $M: I \rightarrow \mathbb{R}$ is multiplicative and the restriction of $M$ to the open interval ] $0,1\left[\right.$ is not identical to $p \mapsto p^{\alpha}$.

Proof. It is obvious that $\left(\mathrm{B}_{1}\right)$ is a solution of $(\overline{F E 3})$. Now consider the case when $g: I \rightarrow \mathbb{R}$ does not vanish identically on $I$. Then there exists an element $q_{0} \in I$ such that $g\left(q_{0}\right) \neq 0$.

Consider the case when $q_{0}=0$. This means $g(0) \neq 0$. Substituting $q=0$ in (FE3) and using $g(0) \neq 0$, solution $g(p)=1-p^{\alpha}$ follows. But this solution is included in $\left(\mathrm{B}_{2}\right)$ with $M=1$. Now consider the situation when $q_{0}=1$. This means $g(1) \neq 0$. Putting $q=1$ in $\left(\right.$ FE3) and using $g(1) \neq 0, g(p)=-p^{\alpha}$ follows. But this solution is also included in $\left(\sqrt{\mathrm{B}_{2}}\right)$ with $M=0$. Now consider the case when $\left.q_{0} \in\right] 0,1\left[\right.$. This means $g\left(q_{0}\right) \neq 0$ but now we must have $g(0)=0$ and $g(1)=0$. Putting $\left.q=q_{0} \in\right] 0,1\left[\right.$ in FE3 and using $g\left(q_{0}\right) \neq 0$, we obtain

$$
p^{\alpha}+g(p)=\left[g\left(q_{0}\right)\right]^{-1}\left[g\left(p q_{0}\right)-q_{0}^{\alpha} g(p)\right]
$$

for all $p \in I$. Define a function $M: I \rightarrow \mathbb{R}$ as

$$
M(x)=\left[g\left(q_{0}\right)\right]^{-1}\left[g\left(x q_{0}\right)-q_{0}^{\alpha} g(x)\right]
$$

for all $x \in I$. Clearly, equation 3.2 gives $M(0)=0$ and $M(1)=1$ as $g(0)=g(1)=0$. Now, from (3.1) and (3.2), (B) follows. From (FE3) and $\left(\mathrm{B}_{2}\right)$, it follows that $M(p q)=M(p) M(q)$ for all $p, q \in I$. Finally, the function $p \rightarrow M(p)-p^{\alpha}$ cannot vanish identically on ]0,1[ because if it is so, then this will mean $M(p)=p^{\alpha}$ for all $p \in I$ because $M(1)=1=1^{\alpha}$ and $M(0)=0=$ $0^{\alpha}$. Consequently, by $\left(\overline{\mathrm{B}_{2}}\right), g(p) \equiv 0$ on $I$ contradicting the assumption that $g: I \rightarrow \mathbb{R}$ does not vanish identically on $I$.

Proof of Theorem 3.1. If $g(p) \equiv 0$, then (FE2 reduces to (1.3). Making use of Result 2.1, the solutions $\left(\mathrm{C}_{1}\right)$ and $\left(\mathrm{C}_{2}\right)$ follow with $\lambda \neq 0$ and $\ell: I \rightarrow \mathbb{R}$ a logarithmic function, which does not vanish identically. From now onwards, we assume that $g$ does not vanish identically on $I$.

Since $f: I \rightarrow \mathbb{R}$ does not vanish identically on $I$, there exists an element $r_{0} \in I$ such that $f\left(r_{0}\right) \neq 0$. 
Let $p, q, r \in I$. Then $f((p q) r)=f(p(q r))$. Now, using FE2 repeatedly,

$$
\begin{aligned}
f((p q) r) & =r^{\beta} f(p q)+(p q)^{\alpha} f(r)+g(p q) f(r) \\
& =r^{\beta}\left\{q^{\beta} f(p)+p^{\alpha} f(q)+g(p) f(q)\right\}+p^{\alpha} q^{\alpha} f(r)+g(p q) f(r) \\
& =r^{\beta} q^{\beta} f(p)+r^{\beta} p^{\alpha} f(q)+p^{\alpha} q^{\alpha} f(r)+r^{\beta} g(p) f(q)+g(p q) f(r) .
\end{aligned}
$$

Similarly

$$
\begin{aligned}
f(p(q r))= & r^{\beta} q^{\beta} f(p)+r^{\beta} p^{\alpha} f(q)+p^{\alpha} q^{\alpha} f(r)+r^{\beta} g(p) f(q) \\
& +p^{\alpha} g(q) f(r)+q^{\alpha} g(p) f(r)+g(p) g(q) f(r) .
\end{aligned}
$$

Equating the expressions for $f((p q) r)$ and $f(p(q r))$ and rearranging the terms, we obtain the equation

$$
f(r)\left[g(p q)-q^{\alpha} g(p)-p^{\alpha} g(q)-g(p) g(q)\right]=0
$$

valid for all $p, q, r \in I$. Choosing $r=r_{0}$ in the above equation and using $f\left(r_{0}\right) \neq 0$, the functional equation (FE3) follows for all $p, q \in I$ but now with $g(p) \not \equiv 0$. By Lemma $3.2, g$ is of the form $\left(\mathrm{B}_{2}\right)$.

Now, from $\left(\mathrm{B}_{2}\right)$ and $(\mathrm{FE} 2)$, we get the equation

$$
f(p q)=q^{\beta} f(p)+M(p) f(q)
$$

valid for all $p, q \in I$ and $M: I \rightarrow \mathbb{R}$ as described in the statement of Lemma 3.2 Since $f(p q)=f(q p)$ for all $p, q \in I, 3.3$ gives rise to the equation

$$
\left[M(q)-q^{\beta}\right] f(p)=\left[M(p)-p^{\beta}\right] f(q)
$$

valid for all $p, q \in I$.

Consider the case when $p \mapsto M(p)-p^{\beta}, p \in I$, vanishes identically on $I$. Then, $M(p)=p^{\beta}$ for all $p \in I$. Hence, $\mathrm{B}_{2}$ gives $g(p)=\left(p^{\beta}-p^{\alpha}\right)$ and also (3.3) reduces to the equation

$$
f(p q)=q^{\beta} f(p)+p^{\beta} f(q)
$$

valid for all $p, q \in I$. By Result 2.1, $f(p)=p^{\beta} \ell(p)$ for all $p \in I, \ell: I \rightarrow \mathbb{R}$ being a logarithmic function, which does not vanish identically and also $0^{\beta} \ell(0)=0$. If $\beta=\alpha$, then $f(p)=p^{\alpha} \ell(p)$ for all $p \in I$ with $0^{\alpha} \ell(0)=0$ and $g(p) \equiv 0$ on $I$ contradicting the fact that $g$ does not vanish identically on $I$. If $\beta \neq \alpha$, then we have $f(p)=p^{\beta} \ell(p)$ for all $p \in I$ with $\beta \neq \alpha$ and $0^{\beta} \ell(0)=0$. Also, now we have $g(p)=\left(p^{\beta}-p^{\alpha}\right), \beta \neq \alpha$. Thus, we have got the solution $\left(\mathbf{C}_{4}\right)$. 
Now consider the case when $p \mapsto M(p)-p^{\beta}, p \in I$, does not vanish identically on $I$. Then there exists an element $\left.p_{0} \in\right] 0,1[$ (as $M(1)=1$ and $M(0)=0)$ such that $\left[M\left(p_{0}\right)-p_{0}^{\beta}\right] \neq 0$. Putting $p=p_{0}$ in 3.4 and using $\left[M\left(p_{0}\right)-p_{0}^{\beta}\right] \neq 0$, it follows that $f(q)=\bar{\mu}\left[M(q)-q^{\beta}\right]$ for all $q \in I$ with $\bar{\mu}=\left[M\left(p_{0}\right)-p_{0}\right]^{-1} f\left(p_{0}\right)$. The case $\bar{\mu}=0$ gives $f(q) \equiv 0$ on $I$, contradicting the assumption that $f: I \rightarrow \mathbb{R}$ does not vanish identically on $I$. Hence, $f(p)=$ $\bar{\mu}\left[M(p)-p^{\beta}\right], \bar{\mu} \neq 0$ being an arbitrary real constant. This, together with $\left(\mathrm{B}_{2}\right)$, constitute the solution $\left(\mathrm{C}_{3}\right)$ of $(\mathrm{FE} 2)$.

Note 3.3. " $f(p) \equiv 0, g$ arbitrary" is a trivial solution of (FE2 irrespective of whether $\alpha=\beta$ or $\alpha \neq \beta$.

Corollary 3.4. Let $c \neq 0$ be a given real constant. If a function $f: I \rightarrow \mathbb{R}$ with $f \neq 0$, satisfies the functional equation (FE1) for all $p, q \in I ; \alpha$ and $\beta$ being positive real exponents which satisfy the conventions stated in $(1.4)$, then the following conclusion holds

$$
\beta=\alpha, \quad f(p)=\frac{1}{c}\left[M(p)-p^{\alpha}\right],
$$

where $M: I \rightarrow \mathbb{R}$ is multiplicative and the restriction of $M$ to the open interval ]0,1[ is not identical to $p \mapsto p^{\alpha}$. In the case when $\beta \neq \alpha$, the functional equation FE1) $(f \neq 0)$ does not have any solution.

Proof. Define $g: I \rightarrow \mathbb{R}$ as

$$
g(x)=c f(x), \quad c \neq 0
$$

for all $x \in I$ and $f \neq 0$. Now (FE2 reduces to the functional equation (FE1), so, in Corollary 3.4, we need to consider only those solutions of (FE2) which satisfies $f \neq 0$. Such solutions are $\left(\mathrm{C}_{3}\right)$ and $\left(\mathrm{C}_{4}\right)$.

Solution $\left(\mathrm{A}_{1}\right)$ of $(\mathrm{FE} 1)$ follows from $\left(\mathrm{C}_{3}\right)$ and $(3.5)$ when $\alpha=\beta$.

From $\left(\mathrm{C}_{4}\right)$ and $(3.5)$, we obtain $f(p)=\frac{1}{c}\left[p^{\beta}-p^{\alpha}\right]$, which satisfies the equation (FE1) only when $\alpha=\beta$. If it is so then $f(p)=0$ which contradicts the fact that $f \neq 0$. Hence, there is only one solution $\mathrm{A}_{1}$ of (FE1) with $f \neq 0$.

Note 3.5. " $f(p) \equiv 0$ " is the only solution of FE1) when $\alpha \neq \beta$.

Note 3.6. There is another generalization of 1.3 , namely,

$$
f(p q)=q^{\beta} f(p)+p^{\alpha} f(q)+\lambda g(p) g(q)
$$


in which $\lambda$ is a given real constant; $p, q \in I ; \alpha$ and $\beta$ are fixed real exponents which satisfy the conventions stated in (1.4); and $f: I \rightarrow \mathbb{R}, g: I \rightarrow \mathbb{R}$ are unknown functions. This functional equation is useful in information theory and statistics when $\alpha=\beta=1$ and $\lambda=2$ (see [9, p. 84]). Due to this reason, it is desirable to study it and some results concerning this will be presented in a subsequent paper.

\section{Measurable solutions of (FE2) and (FE1)}

By a measurable function on $I$ (or a Lebesgue measurable nonempty subset of $I$ ), we shall mean a real-valued function which is measurable in the Lebesgue sense.

By a measurable (or continuous) solution of (FE1), we shall mean a solution $f$ of (FE1) with the property that the function $f$ is measurable (or continuous) on $I$. Likewise, by a measurable (or continuous) solution of (FE2), we shall mean a solution $(f, g)$ (of (FE2) ) with the property that both the functions $f$ and $g$ are measurable (or continuous) on $I$.

If a function $\ell: I \rightarrow \mathbb{R}$ is logarithmic in the sense of Definition 2.2 and is also measurable on $I$ in the Lebesgue sense, then $\ell$ is of the form

$$
\ell(p)= \begin{cases}\bar{\lambda} \log _{2} p & \text { if } 0<p \leq 1, \\ 0 & \text { if } p=0\end{cases}
$$

where $\bar{\lambda}$ is an arbitrary real constant.

The only admissible nonconstant forms of a function $M: I \rightarrow \mathbb{R}$ which is multiplicative in the sense of Definition 2.1 and also measurable on $I$ in the Lebesgue sense are

$$
M(p)= \begin{cases}p^{\delta} & \text { if } 0<p \leq 1 \\ 0 & \text { if } p=0\end{cases}
$$

with $\delta \in \mathbb{R}$ or

$$
M(p)= \begin{cases}0 & \text { if } 0 \leq p<1 \\ 1 & \text { if } p=1\end{cases}
$$


Making use of (4.1), 4.2), (4.3) and Theorem 3.1, the measurable solutions of $(\mathrm{FE} 2)$ are $\left(\mathrm{C}_{1}^{*}\right),\left(\mathrm{C}_{2}^{*}\right),\left(\mathrm{C}_{3 a}\right),\left(\mathrm{C}_{3 b}\right)$ and $\left(\mathrm{C}_{4}^{*}\right)$ where

$$
\begin{aligned}
& \beta \neq \alpha, \quad f(p)=\lambda\left(p^{\alpha}-p^{\beta}\right), \quad g(p) \equiv 0, \\
& \beta=\alpha, \quad f(p)=\bar{\lambda} p^{\alpha} \log _{2} p, \quad g(p) \equiv 0,
\end{aligned}
$$

$\left(\mathrm{C}_{3 a}\right)$

$$
\begin{gathered}
f(p)=\left\{\begin{array}{ll}
\bar{\mu}\left(p^{\delta}-p^{\beta}\right) & \text { if } 0<p \leq 1, \\
0 & \text { if } p=0,
\end{array} \quad g(p)= \begin{cases}\left(p^{\delta}-p^{\alpha}\right) & \text { if } 0<p \leq 1, \\
0 & \text { if } p=0,\end{cases} \right. \\
\left(\mathrm{C}_{3 b}\right) \quad f(p)=\left\{\begin{array}{ll}
-\bar{\mu} p^{\beta} & \text { if } 0 \leq p<1, \\
0 & \text { if } p=1,
\end{array} \quad g(p)= \begin{cases}-p^{\alpha} & \text { if } 0 \leq p<1, \\
0 & \text { if } p=1,\end{cases} \right. \\
\left(\mathrm{C}_{4}^{*}\right) \quad \beta \neq \alpha, \quad f(p)=\bar{\lambda} p^{\beta} \log _{2} p, \quad g(p)=\left(p^{\beta}-p^{\alpha}\right),
\end{gathered}
$$

where $\lambda, \bar{\lambda}$ and $\bar{\mu}$ are arbitrary nonzero real constants; $0^{\alpha} \log _{2} 0:=0$ and $0^{\beta} \log _{2} 0:=0 ; \delta \in \mathbb{R}, \delta \neq \alpha$ and $\delta \neq \beta$.

If $M: I \rightarrow \mathbb{R}$ is a constant multiplicative function, then it is of the form $M(p) \equiv 0$ or $M(p) \equiv 1$. In the former case, the measurable solution $(f, g)$ of (FE2) is

$$
f(p)=-\bar{\mu} p^{\beta}, \quad g(p)=-p^{\alpha} .
$$

In the later case, the measurable solution $(f, g)$ of $(\mathrm{FE} 2)$ is

$$
f(p)=\bar{\mu}\left[1-p^{\beta}\right], \quad g(p)=\left[1-p^{\alpha}\right] .
$$

Likewise, making use of 4.2 , 4.3 and Corollary 3.4 the measurable solutions of (FE1) are as follows:

If $\beta=\alpha$, then the nonconstant measurable solutions of $\left(\right.$ FE1 are $\mathrm{A}_{1 a}$ and $\mathrm{A}_{1 b}$ where

$$
\left(\mathrm{A}_{1 a}\right) \quad f(p)= \begin{cases}\frac{1}{c}\left(p^{\delta}-p^{\alpha}\right) & \text { if } 0<p \leq 1, \\ 0 & \text { if } p=0,\end{cases}
$$

with $\delta \in \mathbb{R}, \delta \neq \alpha$ and

$$
f(p)= \begin{cases}-\frac{1}{c} p^{\alpha} & \text { if } 0 \leq p<1 \\ 0 & \text { if } p=1\end{cases}
$$


In the case of constant multiplicative function $M: I \rightarrow \mathbb{R}$, the measurable solutions of (FE1) are $f(p)=-\frac{1}{c} p^{\alpha}$ when $M \equiv 0$ and $f(p)=\frac{1}{c}\left[1-p^{\alpha}\right]$ when $M \equiv 1$.

Acknowledgement. The authors are grateful to the referee(s) for his valuable suggestions.

\section{References}

[1] Behara M., Additive and Nonadditive Measures of Entropy, Wiley Eastern Limited, New Delhi-Bombay, 1990.

[2] Chaundy T.W., Mcleod J.B., On a functional equation, Edinburgh Math. Notes. 43 (1960), 7-8.

[3] Kannappan PL., Sahoo P.K., On a functional equation connected to sum form nonadditive information measures on an open domain-II, Glas. Mat. 22(42) (1987), 343-351.

[4] Kocsis I., Maksa Gy., The stability of a sum form functional equation arising in information theory, Acta Math. Hungar. 79 (1998), no. 1-2, 39-48.

[5] Losonczi L., Sum form equations on open domain, Utilitas Math. 29 (1986), 125-132.

[6] Losonczi L., Maksa Gy., The general solution of a functional equation in information theory, Glas. Mat. 16(36) (1981), 261-266.

[7] Maksa Gy., Páles Z., Hyperstability of a class of linear functional equations, Acta Math. Paed. Nyire 17 (2001), 107-112.

[8] Nath P., On a functional equation and its relevance in information theory, in: Advances in Information Theory and Operations Research, Om Parkash (Ed.), VDM Verlag, Saarbrucken, Germany, 2010, pp. 1-14.

[9] Nath P., Singh D.K., On a sum form functional equation related to entropies and some moments of a discrete random variable, Demonstratio Math. 42 (2009), no. 1, 83-96.

[10] Shannon C.E., A mathematical theory of communication, Bell. Syst. Tech. Jour. 27 (1948), 348-423; 623-656.

[11] Tabor J., Stability of the Cauchy equation with variable bound, Publ. Math. Debrecen 51 (1997), 165-173.

Department of Mathematics

University of Delhi

DELHI-110007

INDIA

e-mail: pnathmaths@gmail.com
Department of Mathematics

Zakir Husain Delhi College

(UNiversity of Delhi)

JaWAharlal Nehru Marg

DELHI-110002

INDIA

e-mail: dhiraj426@rediffmail.com dksingh@zh.du.ac.in 